\title{
Single Star Scidar first light from Dôme C
}

\author{
Jean Vernin, Merieme Chadid, Eric Aristidi, Max Azouit, Tatiana \\ Sadibekova and Hervé Trinquet
}

LUAN, UMR 6525, Université de Nice - Sophia Antipolis, F-06108 Nice Cedex 1, France email: vernin@unice.fr

\section{Introduction}

In the recent years, a lot of instruments have been put into operation during the polar summer at Dôme C., Then, during the first polar night when the Astro-Concordia sation was open for the first time during winter, about 40 balloons (Azouit \& Vernin (2005)) instrumented to measure optical turbulence profiles and 2 Differencial Image Motion Monitors (DIMM) were setup. The main results from this first important campaign are found in Agabi et al. (2006). It appears from this first night time observations that almost all the optical turbulence was concentrated in the first $30 \mathrm{~m}$ above the ice. At an elevation of $8.5 \mathrm{~m}$ above the ice the seeing is about $1^{\prime \prime} .4$, while above an elevation of $30 \mathrm{~m}$ the seeing drops down to $0^{\prime \prime} .36$. This last figure is coherent with the estimation from Lawrence et al. (2004) if one takes into account that they were not sensitive to the first $30 \mathrm{~m}$., which corresponds to the turbulent surface layer.

For the second winter, we decided to implement the so-called Single Star Scidar (for SSS see Habib et al. 2005, 2006) in order to assess continuously the vertical profiles of both the optical turbulence and the wind speed. Indeed, a balloon gives a cut of the atmosphere with a very good vertical resolution but it traverses optical turbulent layers in few seconds. The SSS technique is able to retreive both $C_{N}^{2}(h)$ and $\mathbf{V}(\mathrm{h})$ vertical profiles from the groud up to $25-30 \mathrm{~km}$ each $15 \mathrm{~s}$, during hours. At Dôme C, and tracking Canopus bright star, it becomes possible to monitor $C_{N}^{2}(h)$ and $\mathbf{V}(\mathrm{h})$ during days almost continuously.

\section{First light}

During spring 2005 began the construction of the Antarctic SSS which was sent to Dôme $\mathrm{C}$ during the fall of the same year. Then the instrument was setup on top of a $8.5 \mathrm{~m}$ high plateform (see Fig. 1) by one of the authors (MC) with the precious help of E. Aristidi, and, on February 4th, we got the first light from this $40 \mathrm{~cm}$ telescope (see <http://www-luan.unice.fr/CHADID/chadid-aristidi.htm>).

Then, night time measurements where performed by E. Aristidi during the whole winter, from March to August. Thousands of profiles were obtained during almost $400 \mathrm{hr}$ of observations. From this huge set of measurements, only a small part have been processed yet in our laboratory, since only few minutes of observations can be sent by e-mail per day.

\section{First profiles}

To imagine what will be the installation of a large telescope at Dôme C, and what will be the consequences of an interaction between such a building and the optical turbulence concentrated in the surface layer, it was of major importance to have the detailed structure of both $C_{N}^{2}(h)$ and $\mathbf{V}(\mathrm{h})$ profiles. But the vertical resolution of the SSS is around one $\mathrm{km}$. Thus we decided to leave the Simulated Annealing method to reconstruct four 




Figure 1. Installation of the Single Star Scidar on a $8.5 \mathrm{~m}$ high plateform at Dôme C during summer 2005.

arbitrary layers at ice level, and the other layers distributed every $1 \mathrm{~km}$. Of course, SSS is not able to distinguish between the altitude of the first four layers, but we assumed that the wind speed is increasing from the bottom to the top of the surface layer, and thus it became possible to sort the four first layers with increasing speed.

As we already know that most of the optical turbulence is concentrated within the first $30 \mathrm{~m}$, and that we are very interested in the $C_{N}^{2}(h)$ and $\mathbf{V}(\mathrm{h})$ profiles within those $30 \mathrm{~m}$, we left the SA algorithm to reconstruct four layers within the SL. In Fig. 2 one can see the rapid decrease of the optical turbulence intensity and the rapid increase of the wind speed. The seeing deduced from the optical turbulence profile is $0^{\prime \prime} .56$, very close to the $0^{\prime \prime} .6-0^{\prime \prime} .7$ measured by the DIMM at the same time.
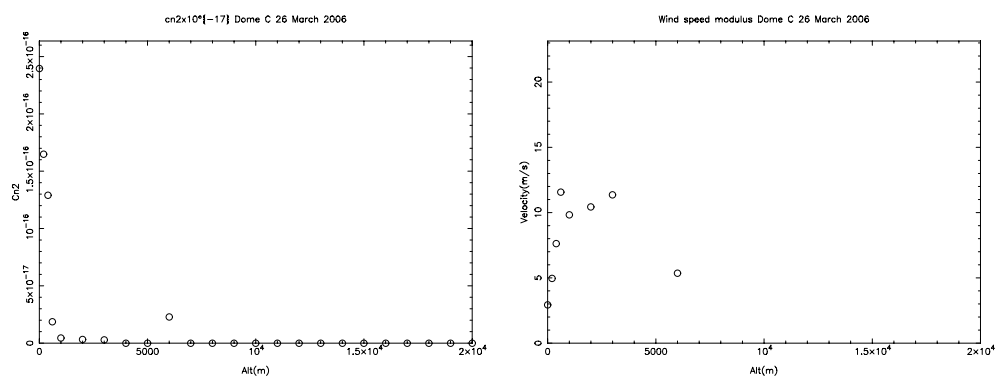

Figure 2. Left: Optical turbulence profile. Rapid decrease of the $C_{N}^{2}$ in the surface layer and a layer at $6 \mathrm{~km}$. Right: Wind speed rapidly increases from 3 to $13 \mathrm{~ms}^{-1}$ in the SL.

\section{Conclusion}

The Antarctica Single Star Scidar was installed at Dôme C during January-February 2006 and ran almost continuously from March to August giving thousands $C_{N}^{2}(h)$ and $\mathbf{V}(\mathrm{h})$ profiles. This will help to have a better knowldge of the optical turbulence within the surface layer as well as the free atmosphere.

\section{References}

Agabi, A., Aristidi, E., Azouit, M., et al. 2006, PASP, 118, 344

Aristidi, E., Agabi, A., Vernin, J., et al. 2003, A $\& A$ (Letters), 406, L19

Azouit, M., \& Vernin, J. 2005, PASP, 117, 536

Habib, A. H., et al. 2005, Comptes Rendus Acad. Sci. Paris, 3, 385

Habib, A., Vernin, J., Benkhaldoun, Z., \& Lanteri, H. 2006, MNRAS, 368, 1456

Lawrence, J. S., Ashley, M. C. B., Tokovinin, A., \& Travouillon, T. 2004, Nature, 431, 278 